\title{
Features of severe asthma in young children from Romania
}

\author{
Mihai Craiu ${ }^{1 *}$, lustina Violeta Stan² \\ From EAACI International Severe Asthma Forum (ISAF 2012) \\ Gothenburg, Sweden. 11-13 October 2012
}

\section{Background and aim}

Asthma is one of the most important chronic disease in children, due to high prevalence and increased direct and indirect costs. A complex effort [GA2LEN, Me-DALL and ARIA groups] was organized to address the area of severe allergic diseases, including severe asthma. The concept of "problematic-severe-asthma" has risen and two major groups of asthmatic children were identified: difficult-totreat asthma and therapy resistant asthma. There were published few papers on asthmatic children from Romania, only one paper addressing the area of severe asthma in school-age children. We describe features of severe asthma in young and preschool children from Romania.

\section{Materials and methods}

Cross-sectional study of patients in tertiary-referral asthma clinic: were included children with previously diagnosed asthma or recurrent-wheezing phenotype, upto 10 -years of age. All these children were referred by GP or pediatrician because of severe/uncontrolled disease. A complex evaluation was performed to exclude alternative diagnostics. At first visit, an extensive training for device use, inhalation technique and trouble-shooting was implemented.

\section{Results}

313 referrals (between Oct 2011-Mar 2012) were evaluated. 233 children were evaluated 1-5 times. 216 children were included. They were 56.7 months old (4-125 mo), $153(70.83 \%)$ were boys, $182(84.26 \%)$ were inner-city children. For 202 (93.53\%) evaluation per-protocol was completed. 153 (75.74\%) had identifiable factors for not achieving control. 49 (24.26\%) had severe disease that generated multiple visits or exacerbations. In 6 children
(12.24\%) asthma was excluded. The remaining 43 (87.76\%) had uncontrolled asthma, but really difficult-totreat or refractory asthma was documented only in 8 (3.7\% of included patients, $16.33 \%$ of children with more severe disease and no identifiable factors). They didn't present male dominance ( $50 \%$ girls) were significantly older (83.6 months) had frequently severe rhino-conjunctivitis (75\%) and atopic dermatitis (62.5\%). In 2 cases severe side effects of medication were documented (severe depression associated with LTRA). High frequency of exacerbations (87.5\% with 1-4 episodes) was noted.

\section{Conclusions}

1. Problematic-severe-asthma is more frequent than previously described in romanian children (3.7\% vs $1.5 \%)$. 2 . They are older, more often girls, have severe allergic associated-diseases and exacerbate more frequent than other asthmatic children in spite of aggressive treatment.

\section{Author details}

${ }^{1}$ Institute for Mother and Child Care, Carol Davila Medical University, MedLife Children's Hospital, Romania. ${ }^{2}$ Institute for Mother and Child Care, Carol Davila Medical University, Respiratory Diseases, Romania.

Published: 3 May 2013

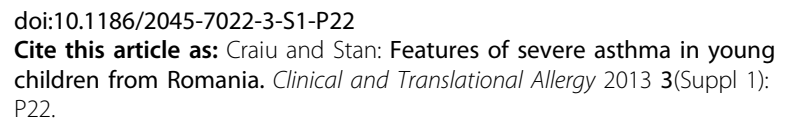

Cite this article as: Craiu and Stan: Features of severe asthma in young children from Romania. Clinical and Translational Allergy 2013 3(Suppl 1): P22. 